\title{
Navigation, world mapping and astrometry with Galileo's moons
}

\author{
Kaare Aksnes \\ Institute of Theoretical Astrophysics \\ University of Oslo, Norway \\ email: kaare.aksnes@astro.uio.no
}

\begin{abstract}
Galileo realized that the four moons he discovered, besides supporting the heliocentric system, could also serve as a clock in the sky for longitude determination. Navigation at sea by this method did not prove practical but G. Cassini used it to improve land mapping. O. Rømer discovered that the interval between eclipses of the moons by Jupiter increased or decreased according to whether the Earth moved away from or toward Jupiter. He attributed this to the finite speed of light which he in 1676 determined with an error of about $25 \%$. Timings of the eclipses by Jupiter have served to compute accurate orbits of the moons, notably by means of R. A. Sampson's theory of 1921. Beginning in 1973, light curves of mutual eclipses and occultations between pairs of moons have been made regularly at six years intervals. From these observations very accurate radii and positions of the moons have been obtained.
\end{abstract}

Keywords. Io, Europa, Ganymede, Callisto, eclipses, ephemerides, occultations, time.

\section{The Galilean clock in the sky}

Galileo discovered the four big satellites of Jupiter on 7 January 1610 and announced his discovery to the Prince of Venice (Figure 1). For the first time Galileo had proof that not all celestial bodies circle around the Earth as the geocentric world view claimed. This convinced him of the correctness of Copernicus' heliocentric theory. Galileo also realized that the satellites provided a clock in the sky which could be seen everywhere and give the longitude of the observer by comparison with local time. At that time chronometers had not yet been invented for determination of longitude at sea, and the available pendulum clocks could not keep accurate time in a rolling ship. It took more than 100 years before the first chronometer was constructed in 1735 by John Harrison.

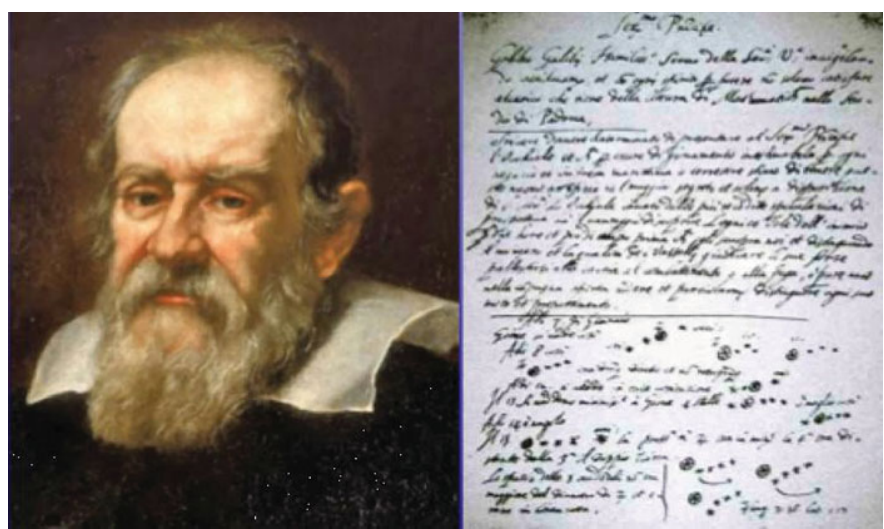

Figure 1. Galileo and his letter to the Prince of Venice. 
In 1612 the Frenchman Nicholas Peiresc tried to read the satellite clock based on the changing locations of the satellites, but they moved too slowly with too poorly known positions for timing. The same year Galileo for the first time observed an eclipse of a satellite by Jupiter or its shadow, and he pointed out that these frequent events could be timed fairly accurately. If one knew an eclipse time at a place of known longitude, the shift in longitude to another place where the eclipse was observed would be equal to the difference between that eclipse time and the local time which could be determined by sightings of the Sun or stars. But Galileo encountered two practical problems: He did not have sufficiently accurate eclipse tables, and the available telescopes needed to observe the satellites had only about 20 arcmin field of view, making it difficult to spot the satellites from an unsteady ship deck. He tried in vain to convince Spanish and Dutch ship navigators to use his technique and he worked for several years on perfecting predictions for the eclipse times.

When Galileo died in 1642, the best available eclipse tables were due to Simon Mayer (Figure 2) in 1614. He claimed to have discovered the four satellites independently of Galileo and at about the same time. But Galileo labeled him an impostor. That was really unjustified and it seems fair that the satellite names Io, Europa, Ganymede and Callisto, originally proposed by Mayer, were finally accepted by The International Astronomical Union in 1973.

Galileo's idea to navigate ships by means of natural satellites did not prove practical. But good ideas never die, and today ship navigation is performed to a very high accuracy by means of artificial satellites in the GPS system.

\section{Giovanni Cassini's land mapping}

It was left to Giovanni Domenico Cassini (1625-1712) (Figure 3), who became the first director of Paris Observatory, to perfect in 1668 the satellite eclipse tables to the extent that they could be used for longitude determination, not at sea but on land. Especially the innermost satellite, Io, with a period of only 1.7 days, lent itself for this purpose. By then, telescopes with convex lenses also offered much larger field of views than the Galileo telescope with a concave eyepiece lens. Cassini led in the 1670s the work to produce a more accurate map of France by means of eclipse observations. The map (Figure 4) was completed in 1679 and showed that the west coast of France on existing maps was a whole degree too far west. This is said to have made Louis XIV exclaim that he was losing more land to his astronomers than to his enemies! The satellite eclipse technique

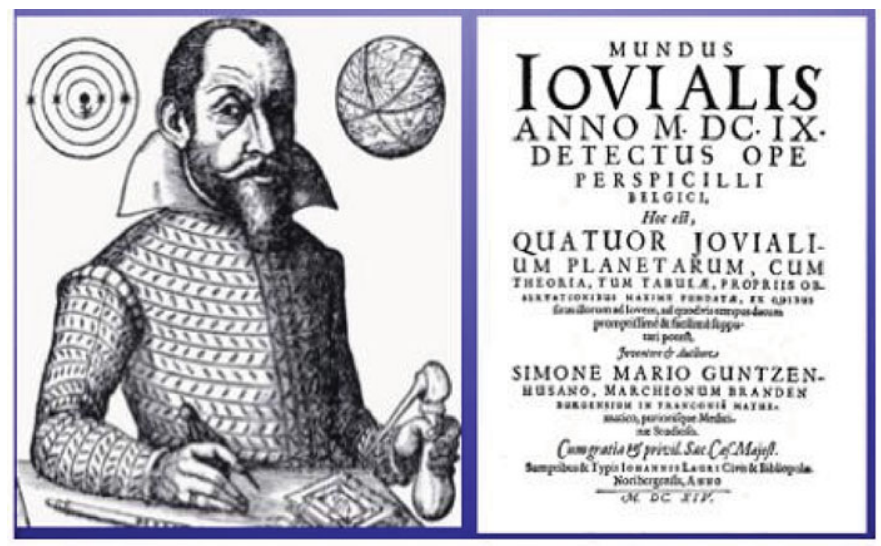

Figure 2. Simon Mayer and his moon tables. 


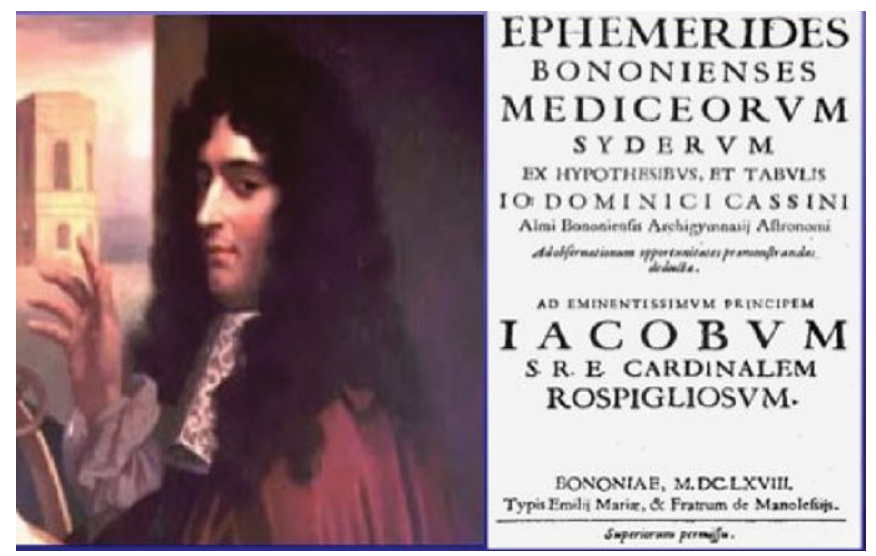

Figure 3. Giovanni Cassini and his moon tables.

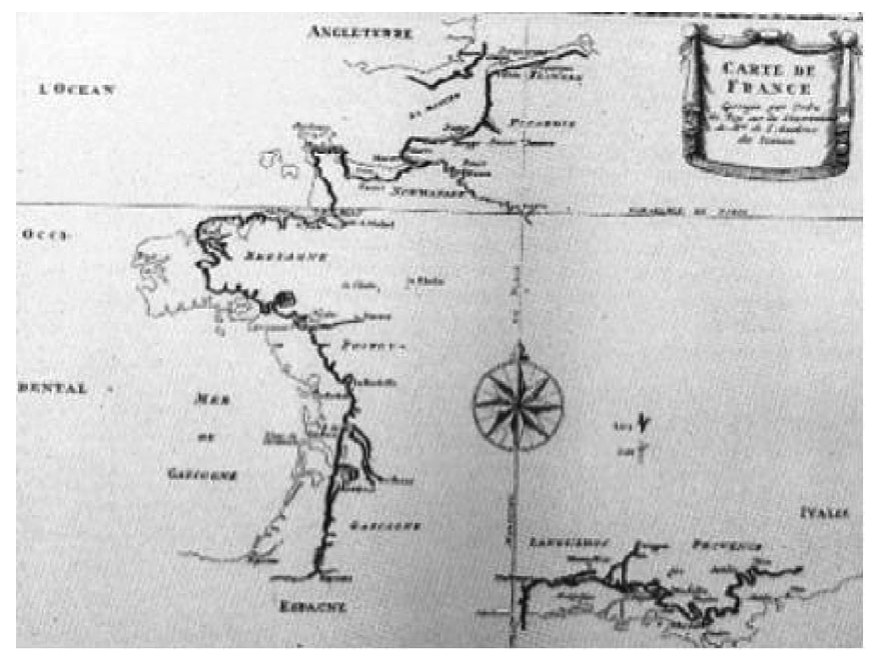

Figure 4. Cassini's map of France.

was even employed in the New World where Charles Mason and Jeremiah Dixon surveyed the border, known as the Dixon line, between Pennsylvania and Maryland.

\section{Ole Rømer measures the speed of light}

Attempts to measure the speed of light by, for instance, observing how long it takes for a light signal to pass from one mountain top to another, were of course doomed to failure. Equipped with Cassini's tables, the Danish astronomer Ole Rømer (1644-1719) (Figure 5) observed the Galilean satellites both in Denmark and at the Paris Observatory under Cassini's guidance. He discovered discrepancies of up to about 10 min between the predicted and observed times of the eclipses. Rømer correctly attributed this to the finite speed of light. The interval between consecutive eclipses will be shortened or lengthened according to whether the Earth in its orbit is moving towards or away from Jupiter. Rømer estimated that the light took 10 to11 min to travel from the Sun to the Earth. The correct figure is $8.3 \mathrm{~min}$, so Rømer's value for the speed of light was about $25 \%$ too low, not bad since the best estimate until then was infinite speed. 


\section{Satellite orbit improvement from eclipses by Jupiter}

It took a long time before a satisfactory theory of motion was developed for the Galilean satellites. Pierre-Simon Laplace (1749-1827) showed (Figure 6) that there are strong interactions between the three inner satellites Io, Europa and Ganymede because their periods or mean motions $n_{1}, n_{2}, n_{3}$ are in the nearly exact ratios 1:2:4. One consequence is that the four satellites can never be seen on the same side of Jupiter.

This gives rise to strong resonances, i.e. oscillations of large amplitudes and very long periods. A satisfactory orbital theory was not available until R. A. Sampson (1921) published a theory based largely on accurate visual timings of the satellites' entry into or exit from Jupiter's shadow (Figure 7). The interactions between the satellites also made it possible to estimate approximate masses of the satellites. Sampson's theory has been improved by Lieske (1987) at JPL, making use of better satellite masses determined from the Voyager flybys of the satellites. Occultations of the satellites by Jupiter's disk, or transits across the disk, cannot be timed as accurately because of the glare of Jupiter. Even for the eclipses, refraction and absorption of sunlight in Jupiter's variable atmosphere introduce considerable uncertainties despite the use of sophisticated models to define the shadow edges. The most accurate way to time the eclipses is by using a photometer to establish light curves of the satellites during shadow entry or exit. The time of eclipse is defined as the instant of half-light. Since 1975 members of the Association of Lunar and Planetary Observers (ALPO) have visually timed more than 10,000 satellite eclipses throughout the world.

\section{Mutual eclipses and occultations among the satellites}

The Galilean satellites move in near-equatorial orbits. Every six years when the orbital planes are seen nearly edge-on from the Earth, the satellites will occult or eclipse one another for periods of more than a year. In such a mutual occultation or eclipse, one satellite or its shadow will be seen to cover another satellite. Although timings of eclipses of the Galilean satellites by Jupiter have provided the bulk of observations for improving the satellites' orbits, the mutual events provide considerably more accurate position data. Because the satellites are airless, the beginning and end of a mutual occultation or eclipse are more precisely defined than is the case for an eclipse by Jupiter. Crude light curves of mutual events were made visually as early as 1926, but accurate light curves were
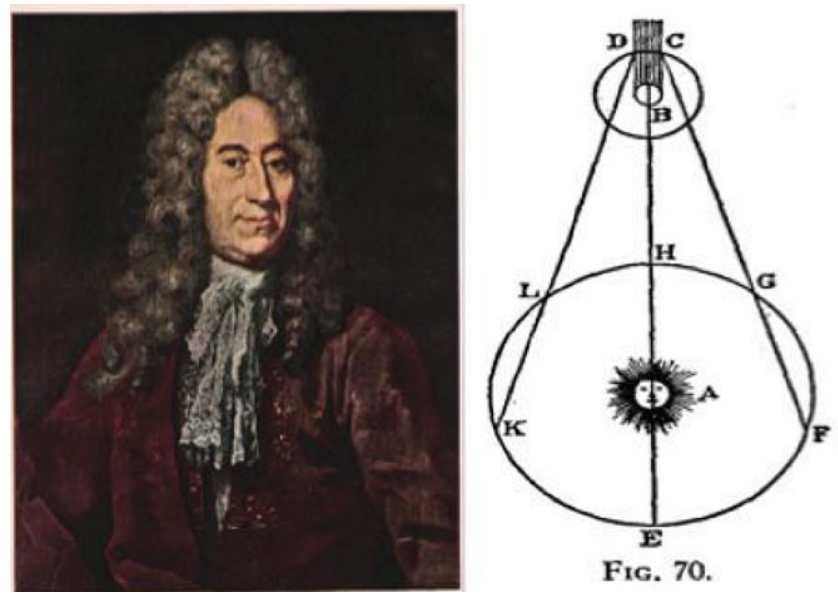

Figure 5. Ole Rømer measures the speed of light. 
first recorded in 1973 when photometers with photomultiplicators had become available. That year a concerted world-wide observing campaign produced many hundred excellent light curves of mutual events.

Figure 8 is an example of an occultation of Europa by Io, that I observed at the Siding Spring Observatory in Australia. Notice the flat bottom of the curve which shows that Io covered Europa totally for about one minute.

In the six observing campaigns carried out world-wide between 1973 and 2003, many thousand observations of mutual satellite events have been recorded. This year there is an on-going seventh observing campaign. Most of the observations have been deposited in a databank (PHEMU) established at the Bureau de Longitude in Paris.

\section{What have we learned from the mutual satellite events?}

Essentially, the observed midtime of a mutual event depends on the longitudes of the satellites in their orbits, while the depth of the light curve depends on the latitudes and on the satellite radii. If $I_{1}$ and $I_{2}$ represent the brightnesses of the two satellites and $A$ is the fraction of satellite 2 occulted by satellite 1 , then the joint brightness during the occultation can be represented as

$$
I=1-A\left(1+I_{1} / I_{2}\right) \quad A=A\left(x, y, R_{1}, R_{2}\right)
$$

The brightness ratio $I_{1} / I_{2}$ can be observed while $A$ can be computed if we know the satellites' relative position $x, y$ from an orbital theory and know the radii $R_{1}$ and $R_{2}$. By making adjustments in the radii and the relative position, the theoretical light curve can be fitted to the observed one through a least squares technique. This way the information of the entire light curve is made use of, and not only the midtime and the depth.

For the eclipses the light curve model is much more complex since one has to compute the light loss in both the umbra and the penumbra.

The radius of Io had been determined very accurately from a star occultation in 1971 giving a mean radius of $1821 \mathrm{~km}$ (O'Leary \& van Flandern 1972). At that time, there was a spread of several hundred kilometers in the radii measured for the other three Galilean satellites with micrometers and radiometers.

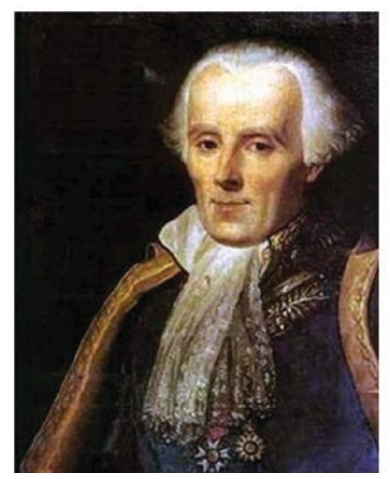

$$
\begin{aligned}
& \mathrm{n}_{1}-2 \mathrm{n}_{2}=\mathrm{n}_{2}-2 \mathrm{n}_{3} \\
& =0.7395 \text { deg/day } \\
& \mathrm{n}_{1}-3 \mathrm{n}_{2}+2 \mathrm{n}_{3}=0 \\
& \mathrm{I}_{1}-3 \mathrm{I}_{2}+2 \mathrm{l}_{3} \approx 180^{\circ} \\
& \mathrm{n}_{1}, \mathrm{n}_{2}, \mathrm{n}_{3}=\text { mean motions } \\
& \mathrm{I}_{1}, \mathrm{l}_{2}, \mathrm{l}_{3}=\text { mean longitudes } \\
& \text { of Io, Europa, Ganymede }
\end{aligned}
$$

Figure 6. Laplace and the Galilean satellite resonances. 
Table 1. Radii $(\mathrm{km})$ of the Galilean moons.

\begin{tabular}{lllll}
\hline Io & Europa & Ganymede & Callisto & Source \\
\hline 1821 & - & - & - & Beta Scorpii occultation 1971 \\
- & 1533 & 2608 & 2445 & 1973 mutual satellite events \\
1822 & 1561 & 2631 & 2410 & Galileo spacecraft 1995-2003 \\
\hline
\end{tabular}

Aksnes \& Franklin (1976) analyzed nearly 100 mutual events observations from 1973 and obtained the mean radii shown in Table 1for Europa, Ganymede and Callisto. These radii differ by at most $30 \mathrm{~km}$ from the more precise values determined later by direct photography from the Pioneer and Voyager spacecraft.

The satellite positions determined from the mutual events have an estimated mean uncertainty of only about 0.03 arcseconds which is an order of magnitude better than what can be obtained from eclipses by Jupiter or Earth based photography.

Just a few days before Voyager 2 arrived at Jupiter in July 1979, Peale, Cassen \& Reynolds (1979) predicted, in an article in Science, that tidal stress in Io should produce volcanism, which was confirmed by dramatic pictures from the spacecraft of many active volcanoes on Io. Io's synchronous rotation and a slight ellipticity forced on Io's orbit by Europa cause the tidal bulge raised by Jupiter to oscillate across Io, flexing its surface. The energy dissipated is taken from Io's orbit which tends to shrink. This is counteracted by the tidal bulge which Io raises on Jupiter and which tries to push Io outward. It has been strongly debated which of these two opposing effects dominates, i.e., whether the time derivative $\mathrm{dn}_{1} / \mathrm{dt}$ of Io's mean motion is positive or negative. Lieske (1987), using mainly eclipse observations covering more than a hundred years, finds a small negative value, while Aksnes \& Franklin (2001), based on analysis of a large number of mutual satellite events, find a bigger positive value, according to which Io is now spiraling slowly

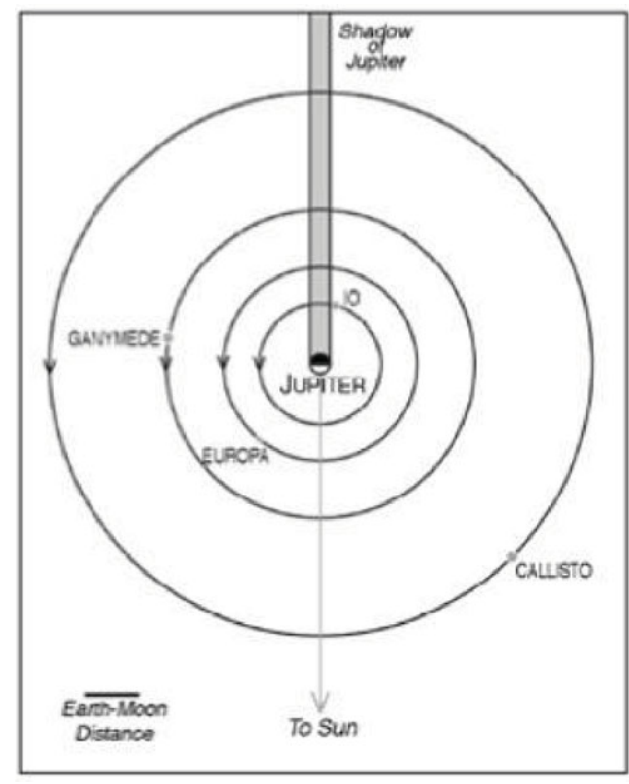

Figure 7. Satellite eclipses by Jupiter. 


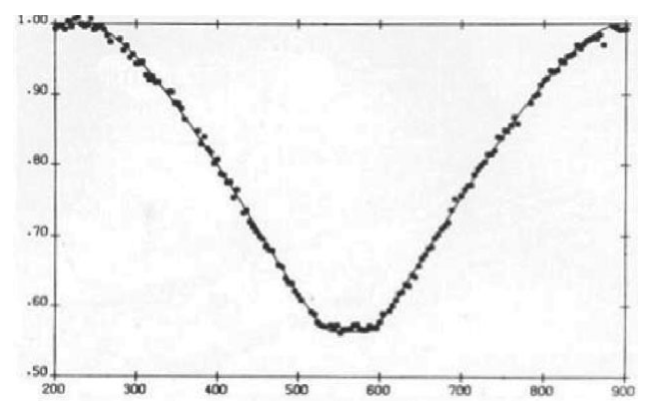

Figure 8. Total occultation of Europa by Io. Time in seconds after 10 September 1973, 12:35 UT.

inward. Recently, Lainey et al. (2009) also find a positive but much smaller value of $\mathrm{dn}_{1} / \mathrm{dt}$, based on tidal modeling and numerical integration over a time span of 116 years. The two last-mentioned articles both conclude that Europa and Ganymede are now moving outward, so that the three inner satellites are moving away from the present Laplacian resonance.

\section{References}

Aksnes, K. \& Franklin, F. A. 1976, AJ, 81, 464

Aksnes, K. \& Franklin, F. A. 2001, AJ, 122, 2734

Lieske, J. H. 1987, A\&A, 176, 146

O’Leary, B. \& van Flandern, T. 1972, Icarus, 17, 209

Sampson, R. A. 1921, Mem. RAS, 63, 1 